\title{
Behavioral functions of the CA3 subregion of the hippocampus
}

\author{
Raymond P. Kesner \\ Department of Psychology, University of Utah, Salt Lake City, Utah 84112, USA
}

\begin{abstract}
From a behavioral perspective, the CA3a,b subregion of the hippocampus plays an important role in the encoding of new spatial information within short-term memory with a duration of seconds and minutes. This can easily be observed in tasks that require rapid encoding, novelty detection, one-trial short-term or working memory, and one-trial cued recall primarily for spatial information. These are tasks that have been assumed to reflect the operations of episodic memory and require interactions between $\mathrm{CA} 3 \mathrm{a}, \mathrm{b}$ and the dentate gyrus via mossy fiber inputs into the CA3a,b. The CA3a,b is also important for encoding of spatial information requiring multiple trials including the acquisition of arbitrary and relational associations. These tasks tend to be non-episodic and can be mediated by arbitrary and conjunctive operations. All these tasks are assumed to operate within an autoassociative network function of the CA3 region. The output from CA3a,b via the fimbria and the medial and lateral perforant path inputs play a supporting role in the neural circuit that supports the operation of these tasks. The CA3a,b also plays a role in sequential processing of information in cooperation with CA1 based on the Schaffer collateral output from CA3a,b to CAl. The CA3a,b also supports retrieval of short-term memory information based on a spatial pattern completion process. Finally, CA3c may, in cooperation with the dentate gyrus, serve an important role in processing the geometry of the environment.
\end{abstract}

This review article emphasizes the importance of behavioral functions of the CA3 subregion of the hippocampus. In the first section, I present a general overview of the anatomy of the hippocampus. In the second section, I present the influence of an autoassociative network function of the CA3 region in supporting mnemonic functions. In general terms, the data suggest that CA3a,b mediates the acquisition and encoding of spatial information within short-term memory with a duration of seconds and minutes. In the context of short-term memory, the CA3a,b mediates rapid encoding of especially spatial information, novelty detection, and one-trial cued recall (all forms of episodic memory). Also, CA3a,b mediates encoding of information requiring multiple trials to construct relational representations. It should be noted that CA3a,b can also be involved in short-term memory retrieval as evidenced by support for a pattern completion process. Finally, CA3c may contribute to pattern separation of the geometry of the environment. Spatial information represents the critical attribute or domain that is processed in CA3. In the third section, I emphasize that the mnemonic functions of the autoassociative network of CA3 depend on inputs into CA3 from other brain regions within the hippocampus (i.e., dentate gyrus) and brain regions outside the hippocampus (i.e., medial and lateral entorhinal cortex) as well as outputs from CA3 to brain regions within the hippocampus (i.e., dentate gyrus, CA1) and brain regions outside the hippocampus (i.e., medial and lateral septum).

\section{Anatomy}

The most prominent anatomical feature of the CA3 subregion is that there are extensive interconnections among the principal cells via a recurrent collateral fiber system (Amaral and Witter 1995). CA3 also receives converging inputs from multiple input pathways; for example, perforant path inputs from the medial and lateral entorhinal cortex, mossy fiber inputs from the dentate gyrus, and its own outputs fed back as inputs via the recur-

E-mail rpkesner@behsci.utah.edu; fax (801) 581-5841.

Article is online at http://www.learnmem.org/cgi/doi/10.1101//m.688207. rent collaterals (Amaral and Witter 1995). In addition to the projections originating in CA1, projections out of Ammon's horn originate in CA3. Many researchers have reported that CA3 projects to the lateral and medial septal nuclei as well as to the vertical limb of the diagonal band of Broca (Gaykema et al. 1991; Amaral and Witter 1995; Risold and Swanson 1997). The medial septum and vertical limb of the diagonal band of Broca, in turn, provide cholinergic and GABAergic inputs into the hippocampus (Amaral and Witter 1995). It has been shown that the CA3 region can be divided into CA3a, b, and c subareas (Lorente de Nó 1934; $\mathrm{Li}$ et al. 1994). A picture of the subdivisions of the CA3 region based on the work of Lorente de Nó is shown in Figure 1. Based on the research of Li et al. (1994) and Buckmaster and Schwartzkroin (1994), it has been proposed that mossy cells receive excitatory inputs from granule cells and CA3c pyramidal cells and integrate the inputs from granule cells and CA3c pyramidal cells, which, in turn, via excitatory recurrent axonal projections activate many distal granule cells. Thus, CA3c may have a backprojection output that can influence the DG granule cells (Scharfman 2007). Most of the synaptic connections embedded in those different pathways in CA3 are modifiable in their strength (Marr 1971; Treves and Rolls 1994). These anatomical and physiological characteristics inspired many theoretical models to assign specific cognitive processes to CA3 (Marr 1971; O'Reilly and McClelland 1994; Treves and Rolls 1994; Hasselmo et al. 1995, 2002; Rolls 1996; Hasselmo and Wyble 1997; Samsonovich and McNaughton 1997; Lisman 1999; Mizumori et al. 1999; Kesner et al. 2004; Rolls and Kesner 2006).

\section{Autoassociative network functions of the $\mathrm{CA} 3$ region}

\section{Rapid encoding of novel information}

One of the mnemonic processes suggested for CA3a,b within short-term memory is the rapid acquisition of novel information. Marr proposed that the hippocampus should be capable of a rapid formation of simple representations, based on modifiable 


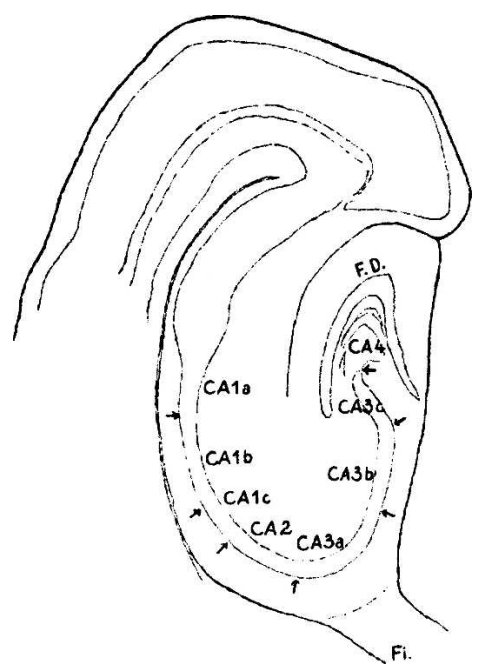

Figure 1. A schematic of the different subregions of the $C A 3$, including CA3a, b, and c, based on drawings from Lorente de Nó (1934).

synaptic recurrent collateral associative connections among its neurons. This idea was further developed by McNaughton and Morris (1987) and Rolls (1989b), who have suggested that, based on CA3 recurrent collateral associative connections, the CA3 system may operate as an attractor network that is useful for some types of working memory. Support for this idea comes from a variety of studies analyzed by Kesner and Rolls (2001) and Rolls and Kesner (2006), including a study by Lee and Kesner (2002), who manipulated the NMDA receptors in CA3a,b by injecting AP5 (a pharmacological blocker for the NMDA receptors) selectively into CA3a,b in a delayed non-matching-to-place (DNMP) task. During a study phase of the task, rats visited a randomly chosen arm in the eight-arm maze and came back to the center platform, where a delay period (10 sec) was imposed in a bucket. After the delay period, the rats faced two adjacent arms on the maze (including the arm that had been visited before the delay period), and the task was to choose the arm that had not been visited during the previous study phase. Rats injected with AP5 were not impaired in performing the DNMP task in the familiar environment in which they had been trained. However, they were initially impaired in performing the task normally when the same task was carried out in a novel environment (i.e., on a novel maze in a novel testing room). AP5 injected into adjacent hippocampal subregions (e.g., DG or CA1) did not produce such deficits in the novel environment. Similar results were obtained with CA3-specific neurotoxic lesions (Lee and Kesner 2003). Nakazawa et al. (2003) also reported similar findings with a mouse strain in which the function of CA3 NMDA receptors was disrupted. These mutant mice were impaired in learning a novel platform location in a modified water maze task, whereas they were normal in finding familiar platform locations. In sum, these results strongly suggest that rapid, plastic changes in the CA3 network are essential for encoding novel information quickly into the hippocampal memory system, and NMDA receptormediated plasticity mechanisms appear to play a significant role in the process.

Additional experimental evidence supporting the role of $\mathrm{CA} 3 \mathrm{a}, \mathrm{b}$ in rapid acquisition of novel information comes from a contextual fear-conditioning experiment with subregion-specific lesions (Lee and Kesner 2004a). In this experiment, rats with CA3a,b-specific neurotoxic lesions were placed in a contextual fear-conditioning chamber (placed in a room with multiple visual landmarks and distinctive odors as contextual cues). The animals were given a 10 -sec tone stimulus footshock that coterminated with a 2-sec footshock. When the freezing response was measured during the intertrial interval period during acquisition (when only contextual cues were available), normal animals exhibited freezing behavior from the beginning. However, rats with CA3a,b lesions displayed a delayed onset of freezing behavior, suggesting that rapid formation of memory for the novel contextual cues was disrupted in these animals. Comparable results were reported by Cravens et al. (2006). They showed that rats with CA3 deletion of NMDA receptors failed to acquire a one-trial context discrimination task when tested $3 \mathrm{~h}$, but not $24 \mathrm{~h}$, after acquisition.

Using a paradigm developed by Poucet (1989), rats with direct infusions of AP5 (an NMDA antagonist) or naloxone (a $\mu$ opiate antagonist) into the CA3a,b were tested for the detection of a novel spatial configuration of familiar objects and the detection of a novel visual object change. The results indicated that naloxone or AP5 infusions into the CA3a,b disrupted novelty detection of both a spatial location and a visual object (Hunsaker et al. 2007c).

Rapid encoding for novel object-place associations has also been shown to be mediated by CA3a,b in that lesions of CA3a,b totally disrupt performance of object-cued recall or place-cued recall tasks (Kesner et al. 2007). More detail is provided in the cued recall section below.

In a different task in which rats were required to remember multiple places, CA3a,b and CA1 lesions produced a deficit. In the task, during the study phase rats were presented with four different places within sections that were sequentially visited by opening of one door to a section at a time on a newly devised maze (i.e., a Tulum maze). Each place was cued by a unique object that was specifically associated with each location within the section during the study phase. Following a 15-sec delay and during the test phase, one door to one section would be opened and in the absence of the cued object in that section, rats were required to recall and revisit the place within that section of the maze that had been previously visited. Both CA1 and CA3a,b lesions disrupted accurate relocation of a previously visited place (Lee et al. 2005). Thus, short-term memory for multiple places in a one-trial multiple place task depends on both CA3a,b and CA1. As an attractor network can generally hold only one item active during a delay period via sustained firing, this type of multiple item short-term memory is computationally predicted to require synaptic modification to store each item, and both CA3a,b and CA1 appear to contribute to this.

Recently, Lee et al. (2004) showed physiologically that the plasticity mechanism in CA3a,b was activated only when animals encountered novel configurations of familiar cues for the first time. Specifically, rats were trained to circle clockwise on a ring track whose surface was composed of four different textural cues (local cues). The ring track was positioned in the center of a curtained area in which various visual landmarks were also available along the curtained walls. To produce a novel cue configuration in the environment, distal landmarks and local cues on the track were rotated in opposite directions (distal landmarks were rotated clockwise, and local cues were rotated counterclockwise by equal amounts). It is well known that principal cells in the hippocampus fire when the animal occupies a certain location of space, known as the "place field" of the cell. Mehta et al. (1997) originally showed that the location of the CA1 place field (measured by the center of mass of the place field) changed over time (shifting backward opposite to the direction of the rat's motion) in a familiar environment as the animal experienced the environment repeatedly. Such a prominent shift in the place field location appears to be associated with the NMDA receptordependent plasticity mechanism in the hippocampus (Ekstrom 
et al. 2001). When the rats encountered the changed cue configurations for the first time in the Lee et al. (2004) experiment, the CA3a,b place fields shifted their locations backward prominently compared to the place fields in CA1. However, such a prominent shift was not observed in CA3a,b from day 2 onward (CA1 place fields started to exhibit a similar property from day 2). This double dissociation in the time course of plasticity between CA1 and CA3a,b place fields suggests that CA3a,b reacts rapidly to any changed components in the environment, presumably to incorporate the novel components into an existing system (or build a new representation of the environment if changes are significant).

Finally, single unit activity has been recorded in CA3 during the delay period in rats in a spatial position short-term memory task (Hampson et al. 2000) and in monkeys in an object-place and a location-scene association short-term memory task (Rolls 1989a; Wirth et al. 2003).

In sum, these results strongly suggest that rapid, plastic changes in the CA3a,b network are essential in encoding novel information involving associations between objects and places, odors and places, or between landmark visual cues and spatial locations, and that NMDA receptor-mediated plasticity mechanisms appear to play a significant role in the process.

\section{Encoding over multiple trials}

The CA3a,b subregion of the hippocampus also appears to be necessary in some tasks that require multiple trials to acquire the task. For example, lesions of the CA3a,b (but not the CA1 or DG) subregion impair the acquisition of object-place and odor-place paired associate learning, a task that requires multiple trials to learn (Gilbert and Kesner 2003). Another multiple trial task in which a learning set is needed that is affected by CA3a,b, but not by CA1, lesions is acquisition of a non-match to sample one-trial spatial location task on an eight-arm maze with 10-sec delays (DNMP) (Lee and Kesner 2003). In this case, the output from $\mathrm{CA} 3 \mathrm{a}, \mathrm{b}$ is via the fimbria, in that fimbria lesions that interrupt the CA3a,b hippocampal output without affecting the input also impair acquisition of this task (Hunsaker et al. 2007a). Another task that requires multiple trials is the Hebb-Williams maze. A pictorial representation of the Hebb-Williams maze is shown in Figure 2. For a detailed analysis of encoding and consolidation/ retrieval processes that may operate in the Hebb-Williams maze, separate indices were developed. It is assumed that encoding and retrieval processes interact on a trial-by-strial basis in any behavioral task. Thus, because it is difficult to discriminate between the contributions of encoding and retrieval for individual trials using a behavioral analysis, encoding and retrieval were given operational definitions based on calculations of trial errors. An encoding index was calculated by subtracting the mean number of errors made per trial during the last five trials of day 1 from the

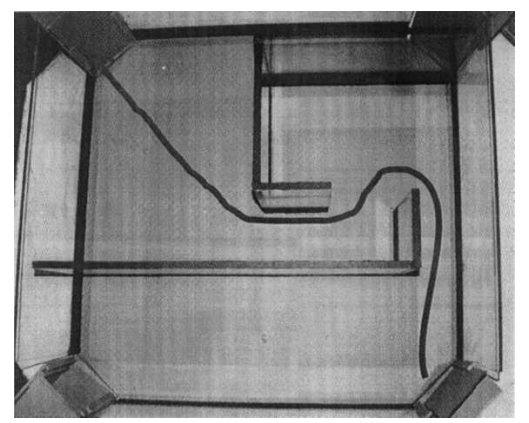

Figure 2. Modified Hebb-Williams Maze paradigm with optimal pathway marked. mean number of errors made during the first five trials of day 1. A retrieval index was calculated by subtracting the mean number of errors made per trial during the first five trials of day 2 from the mean of the last five trials of day 1 . The assumption made in this analysis was that the encoding of spatial information predominated during day 1 , and retrieval of consolidated spatial information predominated during the first five trials during day 2. The results from testing rats in the Hebb-Williams maze indicate that CA3a,b, but not CA1, lesions impair within-day learning (encoding) and CA1, but not CA3a,b, lesions impair retrieval across days (Jerman et al. 2006; Vago et al. 2007). Finally, CA3 lesioned rats are impaired in the standard water maze task, which requires multiple trials (Brun et al. 2002; Florian and Roullet 2004), although mice that lacked NMDA receptors in CA3 do not appear to be impaired in learning the water maze (Nakazawa et al. 2002). These findings are consistent with the hypothesis that when a new environment is learned, this may involve multiple associations between different environmental cues, both with each other and with idiothetic (self-motion) cues, and that this type of learning depends on new associations formed via the CA3-CA3 connections.

It appears that in tasks that require a large number of trials to learn such as the acquisition of the delayed non-matching-toplace task and probably the acquisition of the spatial tasks mentioned above, the object-place learning task, and the HebbWilliams maze task, the CA3a,b outputs via the fimbria are essential, but the outputs to CA1 do not play an important role. The fimbria output would enable CA3a,b to influence the lateral septal area, which could, in turn, influence the medial septum. Any alteration of medial septum functionality would alter cholinergic input to the hippocampus (most of which comes from the medial septum), and this would be expected to attenuate LTP in the recurrent collaterals (Hasselmo et al. 1995), and thus acquisition. Indeed, blockade of CA3 cholinergic receptors with scopolamine disrupts acquisition of the Hebb-Williams maze (Rogers and Kesner 2003), fear conditioning that is specific to the spatial context (Rogers and Kesner 2004), and detection of spatial novelty (Hunsaker et al. 2007c). However, it is possible that septal dysfunction disrupts the responsiveness of hippocampal neurons to environmental inputs, thereby altering attentional processes, which, in turn, could alter memory function (Vinogradova et al. 1996; Hasselmo and McGaughy 2004).

\section{Arbitrary associations}

It has been suggested that the hippocampus and its subregions support the formation of arbitrary associations, including pairedassociate learning (Eichenbaum and Cohen 2001). McNaughton and Morris (1987), Hasselmo and Wyble (1997), and Rolls (1996) have suggested that the hippocampus and specifically the CA3 autoassociative network is responsible for the formation and storage of arbitrary associations. For example, information from the parietal cortex regarding the location of an object may be associated with information from the temporal cortex regarding the identity of an object. These two kinds of information may be projected to the CA3 region of the hippocampus via the medial and lateral perforant path to enable the organism to remember a particular object and its location. Support for this idea comes from the observation that under the influence of direct infusions into the CA3a,b of AP5 (an NMDA antagonist), which should block transmission of spatial information from the medial perforant path, or naloxone (a $\mu$ opiate antagonist), which should block transmission of object information from the lateral perforant path (Breindl et al. 1994), naloxone or AP5 infusions into the CA3a,b disrupted novelty detection of both a spatial location and a visual object (Hunsaker et al. 2007c). 
In order to directly test the involvement of the CA3a,b subregion of the hippocampus in spatial paired-associate learning, rats were trained on a successive discrimination go/no-go task to examine object-place paired-associate learning. In this task, two paired associates were reinforced that consisted of one particular object (A) in one particular location (1) and a different object (B) in a different location (2). Mispairs that were not reinforced included object A in location 2 and object B in location 1. Rats should learn that if an object was presented in its paired location, then the rat should displace the object to receive a reward (Go). However, the rat should withhold displacing the object if it was not in its paired location (No-Go). The results indicate that rats with CA3a,b lesions were impaired in learning object-place paired associations relative to controls (Gilbert and Kesner 2003).

In a second task, rats were trained on a successive discrimination go/no-go task to examine odor-place paired-associate learning. In this task, the same procedure was used, except that rats needed to learn that when an odor was presented in its paired location, the rat should dig in sand mixed with the odor to receive a reward. The results indicated that rats with CA3a,b lesions were severely impaired relative to controls in learning odor-place paired associations (Gilbert and Kesner 2003). However, it should be noted that animals with DG or CA1 lesions learned the object-place or odor-place task as well as controls (Gilbert and Kesner 2003). Similar impairments were obtained following deletion of CA3 NMDA receptors in mice in the acquisition of an odor-context paired-associate learning task (Rajji et al. 2006). These data support the hypothesis that CA3a,b, but not DG or CA1, supports paired-associate learning when a stimulus must be associated with a spatial location. To test this idea further, rats were given CA1, CA3a,b, or control lesions prior to learning an object-trace-odor task. The task was run in a $115-\mathrm{cm}$ linear box in which the rat was presented with an object for 10 sec, after which it was removed, followed by a 10 -sec trace period and by the presentation of an odor $50 \mathrm{~cm}$ away. If the odor and the object were paired, the rat was to dig in the odor cup for a reward. If unpaired, the rat was to refrain from digging. Animals that had CA1 lesions were unable to make the association and never performed above chance, whereas animals that had CA3a,b lesions performed as well as controls (Kesner et al. 2005). These results support the idea that the CA1 is involved in forming arbitrary associations that do not necessarily involve spatial information, as long as temporal processing is required. Thus, the CA1 appears to be critical for mediating associations with temporal components, whereas the CA3a,b is important for mediating associations that involve spatial components. In support of the abovementioned statement, it has been shown that both CA3a,b and CA1 lesions disrupt the acquisition of an object-trace-place paired-associate learning task, suggesting that the CA1 appears to be critical for mediating an association with a temporal component, whereas the CA3a,b is important for an association that involves a spatial component (Hunsaker et al. 2006). Computationally, one hypothesis would be that the CA3 system could provide the working memory necessary for hippocampusdependent associations across time, and that the CA3 then influences the CA1 for this function to be implemented. The actual learning could involve holding one item active in CA3 but continuing firing in an attractor state until the next item in the sequence arrives, when it could be associated with the preceding item by temporally asymmetric synaptic associativity (Rolls and Kesner 2006). The computational suggestion thus is that associations across time could be implemented in the hippocampus by using the same functionality that may be used for sequence memory. This model can account for the CA3a,b and CA1 deficits in the object-trace-place paired-associate learning, but cannot account for the CA1, but not CA3a,b, deficit in the object- trace odor paired associate learning task. A different computational model to explain the role of the hippocampus in mediating arbitrary associations across time is the temporal context model described by Howard et al. (2005) and Howard and Natu (2005). They suggest that the hippocampus binds arbitrary associations supplied by the entorhinal cortex into a retrievable spatiotemporal context. If one assumes that this could occur via the CA3a,b network or via direct connections to CA1, then this model could account for a significant role of CA1 in mediating arbitrary associations across time, including object-trace-odor and object-trace-place associations However, it should be noted that the temporal context model only addresses the function of the hippocampus as a whole and does not address the role of CA1 and its potential underlying network in mediating arbitrary associations across time.

\section{Cued recall}

Even though the studies above measure the acquisition of pairedassociate information, the tasks do not address whether the learning is based on arbitrary associations, in part because the stimuli in the object-place task are integral and cannot be separated from each other. Furthermore, since the tasks are bidirectional discrimination tasks, they are, according to O'Reilly and Rudy (2001) and Morris (2007), likely based on the learning of conjunctions of the cues associated with the task, implying that that the cues cannot easily be separated from each other.

In the standard model (Marr 1971; McNaughton and Morris 1987; Levy 1996; Hasselmo and Wyble 1997; Rolls and Treves 1998; Rolls and Kesner 2006), the CA3 system acts as an autoassociation system. This enables arbitrary (especially spatial in animals and likely language for humans as well) associations to be formed within the hippocampus. The CA3 recurrent collateral associative connections enable bidirectional associations to be formed between whatever stimuli are represented in the hippocampus, in that, for example, any place could be associated with any object, and in that the object could be recalled with a spatial recall cue, or the place with an object recall cue (Rolls and Treves 1998).

In one ingenious experiment, Day et al. (2003) trained rats in a study phase to learn in one trial an association between two flavors of food and two spatial locations. During a recall test phase, they were presented with a flavor that served as a cue for the selection of the correct location. They found that injections of an NMDA receptor antagonist (APV) or AMPA receptor antagonist (CNQX) to the dorsal hippocampus prior to the study phase impaired encoding, but injections of APV prior to the test phase did not impair place recall, whereas injections of CNQX did impair place recall. The interpretation is that, in the hippocampus, NMDA receptors are necessary for forming one-trial flavor-place associations, and that recall can be performed without further involvement of NMDA receptors. The reverse order of cuing the location to recall the flavor has not been tested, so that one cannot be sure whether the hippocampus supports arbitrary associations based on this set of experiments.

Based on the Day et al. (2003) experiment, in the Kesner laboratory a visual object recall for a spatial location task has been developed. In this task, after training to displace objects, rats in the study phase (see Fig. 3A, where each shape represents a different object) are placed in the start box, and when the door in front of the start box is opened, the rats are allowed to displace one object in one location, and then after returning to the start box, the door is opened again, and the rats are allowed to displace a second object in another location. There are 50 possible objects and 48 locations. In the test phase (see Fig. 3A, where the open square represents spatial locations covered by a neutral block), 
$\mathbf{A}$
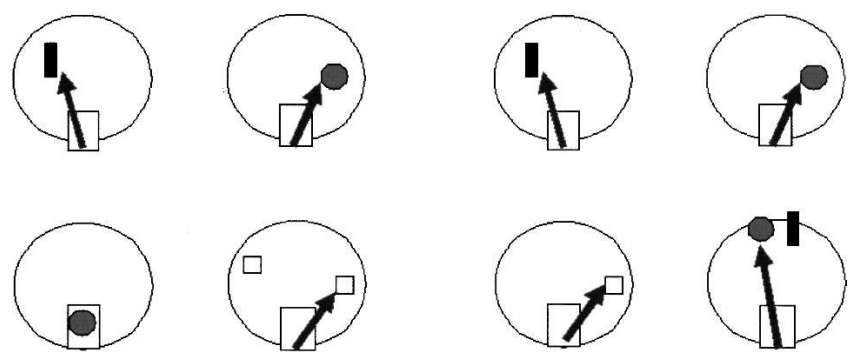

Object Cued Spatial Location

\section{Spatial Location Cued Object}

Figure 3. ( $A$ ) Object-cued spatial location recall. Each shape represents a different object, and the open squares represent spatial locations covered by a neutral block. (B) Spatial location cued object recall. Each shaded shape represents a different object. The open square represents the spatial location covered by a neutral block given as a cue.

the rat is shown one object (first or second randomized) in the start box as a cue, and then, after a 10-sec delay, the door is opened, and the rats must go to the correct location (choosing and displacing one of two identical neutral objects). The rats will receive a reward for selecting the correct location that was associated with the object cue. A spatial location recall for a visual object task has also been developed. For the spatial recall for a visual object task, the study phase (see Fig. 3B, where each shape represents a different object) will be the same, but in this case in the test phase (see Fig. 3B, where the open square represent the location covered by a neutral block given as a cue), when the door is opened, the rat is allowed to displace a neutral object in one location (first or second randomized) on the maze as a location cue, return to the start box, and then, after a 10 -sec delay, the door is opened, and the rats must select the correct object (choosing and displacing one of two visual objects). The rats will receive a reward for selecting the correct visual object that was associated with the location cue. Rats learn both tasks with $\geq 75 \%$ accuracy. Results indicate that CA3a,b lesions produce chance performance on both the one-trial object-place recall and the place-object recall tasks. The potential implications of such results are that, indeed, the CA3a,b supports arbitrary associations as well as episodic memory based on one-trial learning. A control fixed visual conditional to place task with the same delay was not impaired, showing that it is recall after one-trial (or rapid) learning that is impaired (Kesner et al. 2007). Additional support comes from the finding that in a similar one-trial object-place learning followed by recall of the spatial position in which to respond when shown the object, Rolls et al. (2005) showed that some primate hippocampal (including especially CA3a,b) neurons respond with increased activity in the correct spatial location during and after the recall object cue. Thus, some hippocampal neurons appear to reflect spatial recall given an object recall cue. These data are consistent with the prediction of the standard computational model, which emphasizes the importance of CA3a,b in mediating the development of arbitrary associations.

\section{Pattern completion}

During retrieval of information, Marr (1971) suggested that the hippocampus recurrent collaterals should play a major role in the hippocampus in retrieving originally stored information patterns in the face of partial inputs to the hippocampus ("collateral effect" or pattern completion). McNaughton and Morris (1987) and Rolls and Treves (1998) suggested that an autoassociative network within CA3 should be able to support pattern completion. Experimental efforts to find evidence of pattern completion within the CA3 region have been successful in recent years. Support for the pattern completion process in CA3 can be found in lesion studies. In one study, Nakazawa et al. (2002) trained CA3 NMDA receptor-knockout mice in the standard water maze task. When the animals were required to perform the task in an environment where some of the familiar cues were removed, they were impaired in performing the task. The result suggests that the NMDA receptor-dependent synaptic plasticity mechanisms in CA3 are critical to perform the pattern completion process in the hippocampus. In another study (Gold and Kesner 2005), rats were tested on a cheese board with a black curtain with four extramaze cues surrounding the apparatus. (The cheese board is like a dry land water maze with 177 holes on a 119-cm diameter board.) A Plexiglas partition, $7 \mathrm{~cm}$ deep and $8 \mathrm{~cm}$ tall, with a $7-\mathrm{cm}$ opening to permit the animal access to each individual food well, was placed on the tenth row of the cheese board. Rats were trained to move a sample phase object covering a food well that could appear in one of five possible spatial locations. During the test phase of the task, following a 30-sec delay, the animal needs to find the same food well in order to receive reinforcement with the object now removed. Without the Plexiglas partition, rats would adopt a strategy of leaving the goal box to go directly to the center of the maze and make a right or left turn across the line of potential locations of the reward. The Plexiglas partition discouraged rats from using this strategy and forced the animal to make a clear location choice on the test phase by entering the partition surrounding a particular food well. The rats could not see the food until they made a choice. After reaching stable performance in terms of accuracy to find the correct location, rats received lesions in the dorsal CA3a,b. During postsurgery testing, four extramaze cues were always available during the sample phase. However, during the test phase, zero, one, two, or three cues were removed in different combinations. The results indicate that controls performed well on the task regardless of the availability of one, two, three, or all cues, suggesting intact spatial pattern completion. Following the CA3a,b lesion, however, there was an impairment in accuracy compared to the controls, especially when only one or two cues were available, suggesting impairment in spatial pattern completion in CA3a,b lesioned rats (Gold and Kesner 2005). A useful aspect of this task is that the test for the ability to remember a spatial location learned in one presentation can be tested with a varying number of available cues, and many times in which the locations vary, to allow for accurate measurement of pattern completion ability when the information stored on the single presentation must be recalled.

In a different study, Vazdarjanova and Guzowski (2004) placed rats in two different environments separated by $\sim 30 \mathrm{~min}$. The two environments differed greatly in that different objects were located in each room. The investigators were able to monitor the time course of activations of ensembles of neurons in both CA3 and CA1, using a new immediate-early gene-based brain-imaging method (Arc/H1a catFISH). When the two environments were only modestly different, CA3 neurons exhibited higher overlap in their activity between the two environments compared to CA1 neurons. In another study, Lee et al. (2004) recorded from ensembles of neurons using multiple electrodes in both CA1 and CA3a,b in freely behaving animals. By altering cue configuration in a ring track, Lee et al. (2004) demonstrated that the population spatial code in CA3a,b was less disrupted by modestly altered cue configurations. However, the population representation of space in CA1 was more easily disrupted by such moderately altered cue configurations. This can be considered as a pattern completion process in CA3a,b, since the CA3a,b net- 
work maintained similar spatial representation of the environment (compared to the original spatial representation of the familiar cue configurations) even though the relationships among cues in the environment were altered.

Does the dorsal CA3a,b region also play a role in completing a previously learned fixed sequence of spatial locations when the rats are placed in any start position other than the first position and thereby supporting temporal-spatial pattern completion? In one study, Olton et al. (1984) trained rats on fixed sequence in an eight-arm maze based on the magnitude of the reward available in each of four arms $(18,6,1$, or 0 sucrose pellets). After learning the sequence and with some experience of starts in a different arm of the sequence, the rats were given fimbria-fornix or control lesions. The control rats performed the sequence with no difficulty even when new start arms were used, demonstrating temporal-spatial pattern completion. The fimbria-fornix lesioned rats made errors by always returning to the first position in the sequence regardless of which start position was used. This suggests that fimbria-fornix lesioned rats cannot remember the correct temporal-spatial context, but they do not have any difficulty remembering the serial order of the sequence.

In a more recent study, Hoang and Kesner (in press) trained rats in a sequential spatial location list learning paradigm using a correction procedure to visit the arms of an eight-arm maze in a particular sequence, with food obtained in each arm if a correct choice (made by orienting) was made. Even though lesions of the hippocampus or dorsal CA3a,b impaired the acquisition of this task, there were no deficits when hippocampus or dorsal CA3a,b lesions were made after reaching $90 \%-100 \%$ correct performance (DeCoteau and Kesner 2000; R.P. Kesner, in prep.). Thus, the hippocampus or dorsal CA3a,b may be necessary for learning new spatial sequences, but once learned, the hippocampus or dorsal CA3a,b lesioned rats can perform the sequence as long as the rats are started from the beginning of the sequence. To test for subregional specificity, control, dorsal DG, dorsal CA3a,b, and dorsal CA1 lesioned rats were tested postoperatively for completion of the sequence when started in different positions in a previously learned list of spatial locations. The results indicate that control, dorsal CA3a,b, and dorsal CA1 lesioned rats had no difficulty completing the sequence, regardless of starting point. The results also indicate that when the starting position is changed, control and dorsal DG lesioned rats can successfully complete the sequence from any item in the sequence. In contrast, when the dorsal CA3a,b lesioned rats were started in different positions within the sequence, they would invariably orient in front of the first position before making other response sequence errors. On a quantitative level when they made an orienting response to the wrong door (error) in the sequence, the average probability of making incorrect orienting responses for each position indicated that the dorsal CA3 rats made many incorrect orienting responses (errors). This suggests that CA3a,b lesioned rats cannot remember the correct temporal-spatial context, implying difficulty in temporal-spatial pattern completion. These data are consistent with the observation that fimbriafornix lesioned rats always returned to the beginning of the first position in the sequence regardless of which start position was used (Olton et al. 1984), even though it should be noted that in the Olton study, the list length was shorter, the list sequence was influenced by magnitude of reinforcement, and the lesioned rats completed the sequence without making many errors. In the same study, when the dorsal CA1 lesioned rats were started in different positions within the sequence, they would orient in front of any of the forward located positions, suggesting the use of random orienting responses (errors) across the completion of the sequence. On a quantitative level when they made an orienting response to the wrong door (error) in the sequence, the average probability of making an incorrect orienting response indicated that CA1 lesioned rats made decreasing numbers of incorrect orienting responses as the starting position was increased. The errors made by CA1 lesioned rats appeared to be due to difficulty in temporal-spatial pattern completion in that the decrease in errors was due to the observation that all the errors were made in the process of completing the spatio-temporal sequence, suggesting that CA1 lesioned rats have difficulty in remembering the serial order of the spatial sequence. These results suggest that the dorsal CA3a,b in conjunction with CA1 supports recall of a temporal sequence of spatial locations, requiring a completion process. Both CA3a,b and CA1 are likely involved because CA3a,b processes the spatial-temporal context and CA1 processes temporal information. From a computational point of view, it has been suggested that temporally asymmetric associations between the successive pairs of items in a sequence are implemented in dorsal CA3 by some temporally asymmetric synaptic modifiability (Levy 1996; Mehta et al. 2002; Howard et al. 2005; Jensen and Lisman 2005), and the dorsal CA1 network in this situation could help to separate out the representation of the next item in the list from the currently processed item via a chunking process and thus providing for a temporal-spatial context, suggesting that the dorsal CA1 can be thought of as performing temporal pattern completion for spatial locations (Kesner et al. 2004; Rolls and Kesner 2006). These models are consistent with the evidence that rats with hippocampus or dorsal CA3 lesions do not learn the fixed spatial sequence task (DeCoteau and Kesner 2000; R.P. Kesner and M.R. Hunsaker, in prep.). Alternatively, Lisman (1999), Lisman and Otmakhova (2001), and Lisman and Grace (2005) have suggested that the hippocampus operates a match-mismatch detector during the processing of novel changes within a sequence or start of a sequence. They suggest that the first spatial location can serve as a trigger for recall of the entire sequence within the dorsal CA3 region, which would be consistent with the errors made by CA3a,b lesioned rats that involved an error for the first position regardless of which start position was used. Because the readout is presumed to be time-compressed, it reaches dorsal CA1 more quickly, allowing the dorsal CA1 region to anticipate what will happen next based on previous experience. Based on the idea that the dorsal CA1 operates as a match-mismatch detector comparing predictions arriving from dorsal CA3a,b compared to direct entorhinal inputs into dorsal CA1 (Hasselmo and Schnell 1994; Hasselmo and Wyble 1997), the dorsal CA1 should play an important role in detection of changes in the sequence, which is consistent with the observation that lesions of the dorsal CA1 region result in errors that appeared to be random. Thus, the hippocampus and, more specifically, the dorsal CA1 and dorsal CA3a,b subregions contribute to the processing of previously learned sequence information for spatial locations and most likely contribute to the establishment of learned sequences.

\section{Short-term memory}

Finally, the CA3 network also seems essential in supporting the retrieval of information from memory when a short-term delay (e.g., $10 \mathrm{sec}$ ) is introduced (Kesner and Rolls 2001; Rolls and Kesner 2006; Kesner et al. 2007). As the information circulates through the recurrent network in CA3, buffering of information within the network is likely to occur. Owing to such holding phenomenon for neural activities within the recurrent network, CA3 may play a key role in short-term memory tasks, especially when the nature of the tasks entails encoding of novel information or pattern completion. Most experimental results previously described here support this short-term memory function of CA3, since a short-term delay period was imposed in those tasks before 
the animals made choices. When the task prolonged the delay significantly exceeding the short-term range (e.g., $5 \mathrm{~min}$ or $24 \mathrm{~h}$ ), CA3a,b did not remain as a key player in retrieving memory because after the increased delay CA1 became more important (Lee and Kesner 2003).

\section{Input pathways into $C A 3 a, b, c:$ Mossy fiber versus direct perforant path}

McNaughton and Morris (1987), Rolls and Treves (1998), and Rolls and Kesner (2006) have suggested that the dentate granule cell/mossy fiber pathway to CA3 may be important during the learning of new associations in the CA3 network, and that part of the way in which it is important is that it helps by pattern separation to produce relatively sparse and orthogonal representations in CA3. In contrast, the theory predicts that the direct perforant path input to CA3 is important in initiating retrieval from the CA3 autoassociation network, especially with an incomplete retrieval cue.

Support for this hypothesis comes from the findings that Lee and Kesner (2004b) and Jerman et al. (2006) have shown that lesions of the DG or CA3a,b (or a crossed lesion) disrupt withinday learning on the Hebb-Williams maze, but that retrieval of information at the start of the following day is not impaired. In contrast, lesions of the perforant path input to CA3a,b from entorhinal cortex disrupt retrieval (i.e., initial performance on the following day), but not learning within a day (Lee and Kesner 2004b).

The perforant path can be divided into a medial and lateral component. It has been suggested that the medial component processes spatial information and that the lateral component processes nonspatial (e.g., objects, odors) information (Witter et al. 1989; Hargreaves et al. 2005). In one study, Ferbinteanu et al. (1999) showed that lesions of the medial perforant path disrupted water maze learning, whereas lateral perforant path lesions had no effect. In a more recent study based on the idea that the medial perforant path (MPP) input into the CA3 mediates spatial information via activation of NMDA receptors and the lateral perforant path (LPP) input into the CA3 mediates visual object information via activation of opioid receptors, the following experiment was conducted. Using a paradigm developed by Poucet (1989), rats were tested for the detection of a novel spatial change and the detection of a novel visual object change under the influence of direct infusions of AP5 (an NMDA antagonist) or naloxone (a $\mu$ opiate antagonist) into the CA3a,b. The results indicated that naloxone or AP5 infusions into the CA3a,b disrupted both novelty detection of a spatial location and a visual object (Hunsaker et al. 2007c). Based on electrophysiological data, there is associative LTP between the medial or lateral perforant path and the intrinsic commissural/associational-CA3 synapses, demonstrated by the finding of an associative (cooperative) LTP between the medial and lateral perforant path inputs to the CA3 neurons (Martinez et al. 2002). This could provide a mechanism for object (via LPP)-place (via MPP) associative learning, with either the object or the place during recall activating a CA3 neuron. Either place or object recall cues could thus be introduced by the associative MPP and LPP connections to CA3 cells. This is consistent with the finding that disruption of the perforant path input impairs the multiple trial acquisition of an object-place paired-associate task (I. Lee and R.P. Kesner, unpubl.).

It should be noted that in contrast to the results obtained with a CA3a,b injection of naloxone or AP5, naloxone infusions into the DG disrupted both novelty detection of a spatial location and a visual object, whereas AP5 infusions into the DG disrupted only detection of a novel spatial location, but had no effect on detection of a novel object. Furthermore, infusions of AP5 into the CA1 region disrupt only the detection of a spatial location change, but not a visual object change, whereas naloxone into the CA1 region disrupts only the detection of a visual object change, but not a spatial location change (Krug et al. 2001; Hunsaker et al. 2007c). Thus, input pathways from the medial and lateral perforant path have a different effect on each subregion, in part based on the computational processes carried out by that specific subregion.

It is important to note that disruption of DG and mossy fiber inputs into CA3a,b do not produce a disruption in the acquisition of the object-place paired-associate task (Gilbert and Kesner 2003) unless the stimuli are close together, implying that the DG contribution is important particularly when pattern separation is needed (P.E. Gilbert and R.P. Kesner, unpubl.). The implication is that sufficient input for object-place learning can be introduced into the CA3 system (which is required for this object-place learning) by the perforant path inputs provided that spatial pattern separation is not at a premium.

Furthermore, with respect to short-term memory, the DG and mossy fiber input into CA3 are not directly involved. This is illustrated by the observation that on a spatial pattern separation task, the performance of DG lesioned rats increased as a function of increased spatial separation between the target location and the foil location, whereas CA3 lesions were impaired across all spatial separations, indicating a potential impairment in shortterm memory for spatial location information (Gilbert et al. 2001; Gilbert and Kesner 2006).

Thus, from an anatomical perspective, possible dissociations between DG and mossy fiber input into CA3 are primarily due to the observation that the CA3 subregion of the hippocampus has two major inputs with a direct connection from the DG via the mossy fibers and a direct input from the perforant path that bypasses the DG. In addition, short-term memory may be generated intrinsically in the CA3 region, but not in the DG.

\section{Output pathways from CA3a,b,c}

\section{Output from CA3a,b to septum: Fimbria}

One type of result that has been obtained when one compares the relationships between CA3a,b and CA1 is that there is a deficit following dysfunction of the CA3a,b subregion, but no concomitant deficit following dysfunction of the CA1 subregion. For example, lesions of the CA3a,b, but not the CA1, subregion impair the acquisition of object-place and odor-place pairedassociate learning (Gilbert and Kesner 2003), and acquisition of the DNMP task on an eight-arm maze with 10-sec delays (Lee and Kesner 2003). Furthermore, CA3a,b, but not CA1, lesions impair within-day learning (encoding) in a Hebb-Williams maze (Jerman et al. 2006; Vago et al. 2007). Given that CA1 represents the primary output from the hippocampus, especially in light of the fact that CA3a,b does not have direct axonal projections to the subiculum or entorhinal cortex, how can information be transmitted to other neural regions outside the hippocampus once CA1 is ablated? This is very important since other studies have also reported a lack or transient deficit in spatial memory following selective damage to CA1 (Mizumori et al. 1990). One possibility is that outputs that originate from CA3 project via the fimbria directly to the vertical limb of the diagonal band of Broca and medial septum (MS) or indirectly via the lateral septum to MS (Raisman et al. 1966; Swanson and Cowan 1977, 1979; Wyss et al. 1980; Gaykema et al. 1991). The vertical limb of the diagonal band of Broca and MS, in turn, provides cholinergic and GABAergic inputs back to the hippocampus, especially the CA3 subregion. These connections have been characterized anatomically via degeneration, fluorescent tracer, and immunohisto- 
chemical experiments (Raisman et al. 1966; Swanson and Cowan 1977, 1979; Wyss et al. 1980; Gaykema et al. 1991). The connections via the fimbria to the vertical limb of the diagonal band of Broca and MS and especially onto the cholinergic neurons provide an anatomical locus for modulating cholinergic inputs into the hippocampus and especially CA3. An alternate output pathway from the MS to the hippocampus is provided by projections to subiculum and entorhinal cortex, as well as the mammillary bodies. In turn, the mammillary bodies project to the anterior thalamus, which projects to the entorhinal cortex via the preand para-subiculum (Vann and Aggleton 2004). There are, therefore, multiple pathways whereby the outputs from CA3 in the fimbria can send information to the entorhinal cortex and subsequently the hippocampus.

In order to examine the hypothesis that the CA3 efferents via the fimbria are involved in acquiring tasks that require learning over multiple trials, the ventrolateral half of the fimbria was transected under electrophysiological control with a retractable wire knife. The rats were then tested in the acquisition of a nonmatch to sample one-trial spatial location task on an eight-arm maze with 10-sec delays.

Results indicate that fimbria lesioned rats are impaired in acquiring the task similar to CA3a,b lesioned rats. Furthermore, pairing a CA1 lesion with a fimbria transection mimics the effect of a CA3a,b lesion, but with longer-lasting deficits. In addition, neurophysiological and acetylcholinesterase and BDA (an anterograde tracer) results suggest that the output from dorsal CA3 to the medial septum via the fimbria was damaged without removing the input connections back to dorsal CA3a,b (Hunsaker et al. 2007a). Furthermore, the same fimbria transection also mimics CA3a,b lesions in that there is a deficit in the encoding associated with within-day learning, but not between-day retrieval, in the Hebb-Williams maze as well as an impairment in detection of spatial novelty (Hunsaker et al. 2007b; M.R. Hunsaker et al., in prep.).

One explanation that could provide a mechanism for understanding the fimbria cut induced acquisition deficit is based on the importance of the MS to CA3 connection (Swanson and Cowan 1979), since even a small disruption to the modulatory cholinergic system could disrupt the ability of CA3 neurons to encode new information (Hasselmo et al. 1995). It is assumed that the fimbria output to the MS is excitatory, so that a cut in the fimbria output would result in reduced activation of cholinergic fiber projections, especially to CA3. The consequence of a reduction in the cholinergic influence on the hippocampus could be an increase in the effective strength of the reciprocal, recurrent CA3 connections, which would impair new learning because existing associations in the CA3 to CA3 network would tend to dominate the CA3 cell firing (Hasselmo et al. 1995). In this sense, low acetylcholine is appropriate for recall but not for new learning; with low acetylcholine, memories already in CA3 may interfere with setting up new representations in CA3 to be learned. In addition, low acetylcholine after a fimbria cut could impair new learning because long-term potentiation in the CA3to-CA3 synapses is reduced (Hasselmo et al. 1995). These acquisition deficits may be especially evident when the task is difficult to learn and requires multiple trials, and interference in encoding of new spatial information becomes more pronounced. Consistent with these hypotheses are observations of Rogers and Kesner $(2003,2004)$ in which they examined the effects of scopolamine injections into dorsal CA3 on the acquisition of the modified Hebb-Williams maze as well as the acquisition of contextual delay fear conditioning. Scopolamine acted to inhibit or reduce encoding or acquisition over multiple trials but had no real effect on retrieval. The same encoding/retrieval effect was observed for spatial contextual fear conditioning (Rogers and Kesner 2003). In another study, Pereira et al. (2005) showed that injections of 192 IgG-saporin in the hippocampus, which produced a severe depletion of cholinergic cells in the MS, was sufficient to disrupt the acquisition of the standard Hebb-Williams maze. These data also fit with modeling data indicating that MS innervates CA3 more strongly than CA1 (Hasselmo et al. 1995), which again emphasizes the role of CA3 in encoding. Another possibility that needs to be considered involves the output route from MS that projects directly to the subiculum and entorhinal cortex or directly to the mammillary bodies and then via the anterior thalamus and preand para-subiculum to the entorhinal cortex and then hippocampus (Vann and Aggleton 2004).

\section{Output pathways from CA3a,b,c to CAl: Schaffer collaterals}

One can observe a deficit following dysfunction of either the CA1 or CA3 subregion in a specific set of tasks associated with temporal processing, suggesting an important influence of CA3 output into CA1. For example, for temporal pattern separation for spatial locations and for temporal pattern completion for spatial locations, there are deficits following CA1 or CA3a,b lesions (Gilbert et al. 2001; Hoang and Kesner, in press; P.E. Gilbert and R.P. Kesner, in prep.). Thus, for certain types of temporal processing, there is an implication that both regions are working cooperatively and that CA1 is likely to benefit from a feed-forward Schaffer collateral connection from CA3a,b, but CA1 could also be receiving critical information from the direct perforant path.

\section{Output pathways from CA3c to dentate gyrus}

It has been suggested that CA3 might also play a role in spatial pattern separation, but in this case not for a metric spatial representation, but for spatial representation of the geometry of the environment. This idea is supported by the finding of Tanila (1999), who showed that CA3c place cells were able to maintain distinct representations of two visually identical environments, and selectively reactivate either one of the representation patterns depending on the experience of the rat. Also, Leutgeb et al. $(2005,2007)$ recently showed that when rats experienced a completely different environment, CA3c place cells developed orthogonal representations of those different environments by changing their firing rates between the two environments, whereas CA1 place cells maintained similar responses. One model described by Rolls and Kesner (2006) to account for these results proposes that the CA3 and DG may have similar representations of the environment. This may be consistent with the computational point of view that if dorsal CA3 is an autoassociator, the pattern representations in it should be as orthogonal as possible to maximize memory capacity and minimize interference. The theory holds that the actual pattern separation may be performed as a result of the operation of the dentate granule cells as a competitive net, and the nature of the mossy fiber connections to dorsal CA3 cells. To test this idea, an experiment was conducted to determine whether the DG or CA3 regions cooperate to perform spatial pattern separation operations for specific spatial locations as well as the spatial geometry of the environment or whether the DG performs spatial pattern separation on the basis of specific locations in space and the CA3 performs spatial pattern separation on the basis of the geometry of the environment. Rats with lesions of DG and CA3a,b were given the opportunity to explore a white or black circular or square box of the same size as reported by Leutgeb et al. (2007), and in addition, in the box there were two objects spaced $68 \mathrm{~cm}$ apart. After habituation to the box and the objects, the rats received one of three transfer tests. In the first test, the objects were changed to 
a 38-cm distance, but the box shape (geometry of the environment) remained the same. In the second test, the box shape (geometric environment) was changed, but the distance between the objects remained the same; and in the third test, both the shape of the box and the distance between the objects were changed. The efficacy of the transfer test in terms of reexploration of the metric change is based on a comparison between the level of object exploration during the transfer session versus object exploration during the last session of habituation. Similarly, the efficacy of the transfer test in terms of reexploration of the geometry of the environment is based on the number of grid crossings (activity level) and rearings during the transfer session versus the number of grid crossings and rearings during the last session of habituation. The results indicate that lesions of the DG, but not CA3a,b, disrupt both the detection of metric changes in the spatial location of objects and changes in a geometrical environment. Thus far, these data are consistent with the prediction of the Rolls computational model that the DG is the critical substrate for spatial pattern separation. These data are not consistent with the findings of Tanila (1999) and Leutgeb et al. $(2005,2007)$ of a pattern separation function for geometrical environments. It has been shown that the CA3 region can be divided into CA3a, b, and c subareas (Lorente de Nó 1934; Li et al. 1994). Most of the recording of cells that respond to different environments reported by Tanila (1999) and Leutgeb et al. $(2005,2007)$ were based on electrode placements in the $\mathrm{CA3c}$ area. The present lesion data are based on lesions within $\mathrm{CA3a} / \mathrm{b}$, but not CA3c. Based on the research of Li et al. (1994) and Buckmaster and Schwartzkroin (1994), it has been proposed that mossy cells receive excitatory inputs from granule cells and CA3c pyramidal cells and integrate the inputs from granule cells and CA3c pyramidal cells, which, in turn, activate many distal granule cells via excitatory recurrent axonal projections. Such a circuit could integrate spatial location information and form representations of geometrical environments. Additional experiments with CA3c lesions in contrast to the CA3a/b lesions are being carried out. Thus, it is possible that one of the functions of CA3c output back to the granule cells of DG is to influence the detection of spatial pattern separation of the geometry of the environment.

\section{Conclusions}

From a behavioral perspective, the CA3a,b subregion of the hippocampus plays an important role in the encoding of new spatial information within short-term memory with a duration of seconds and minutes. This can easily be observed in tasks that require rapid encoding, novelty detection, one-trial short-term or working memory, and one-trial cued recall primarily for spatial information. These are tasks that have been assumed to reflect the operations of episodic memory and require interactions between CA3a,b and the dentate gyrus via mossy fiber inputs into the CA3a,b. The CA3a,b is also important for encoding of spatial information requiring multiple trials including the acquisition of arbitrary associations. These tasks tend to be non-episodic and mediated by arbitrary and conjunctive operations. All these tasks are assumed to operate within an autoassociative network function of the CA3 region. The output from CA3a,b via the fimbria and the medial and lateral perforant path inputs plays a supporting role in the neural circuit that supports the operation of these tasks. The CA3a,b also supports sequential processing of information in cooperation with CA1 based on the Schaffer collateral output from CA3a,b to CA1. The CA3a,b also plays an important role in retrieval of short-term memory information based on a spatial pattern completion process. Finally, CA3a,b may, in cooperation with the dentate gyrus, play an important role in processing the geometry of the environment.

\section{References}

Amaral, D.G. and Witter, M.P. 1995. The hippocampal formation. In The rat nervous system (ed. G. Paxinos), pp. 443-493. Academic Press, San Diego.

Breindl, A., Derrick, B.E., Rodriguez, S.B., and Martinez Jr., J.L. 1994. Opioid receptor-dependent long-term potentiation at the lateral perforant path-CA3 synapse in rat hippocampus. Brain Res. Bull. 33: $17-24$.

Brun, V.H., Otnass, M.K., Molden, S., Steffenach, H.A., Witter, M.P., Moser, M.B., and Moser, E.I. 2002. Place cells and place recognition maintained by direct entorhinal-hippocampal circuitry. Science 296: $2243-2246$.

Buckmaster, P.S. and Schwartzkroin, P.A. 1994. Hippocampal mossy cell function: A speculative view. Hippocampus 4: 393-402.

Cravens, C.J., Vargas-Pinto, N., Christian, K.M., and Nakazawa, K. 2006. CA3 NMDA receptors are crucial for rapid and automatic representation of context memory. Eur. J. Neurosci. 24: 1771-1780.

Day, M., Langston, R., and Morris, R.G. 2003. Glutamate-receptor-mediated encoding and retrieval of paired-associate learning. Nature 424: 205-209.

DeCoteau, W.E. and Kesner, R.P. 2000. A double dissociation between the rat hippocampus and medial caudoputamen in processing two forms of knowledge. Behav. Neurosci. 114: 1096-1108.

Eichenbaum, H. and Cohen, N.J. 2001. From conditioning to conscious recollection: Memory systems of the brain. Oxford University Press, New York.

Ekstrom, A.D., Meltzer, J., McNaughton, B.L., and Barnes, C.A. 2001. NMDA receptor antagonism blocks experience-dependent expansion of hippocampal "place fields." Neuron 31: 631-638.

Ferbinteanu, J., Holsinger, R.M., and McDonald, R.J. 1999. Lesions of the medial or lateral perforant path have different effects on hippocampal contributions to place learning and on fear conditioning to context. Behav. Brain Res. 101: $65-84$

Florian, C. and Roullet, P. 2004. Hippocampal CA3-region is crucial for acquisition and memory consolidation in Morris water maze task in mice. Behav. Brain Res. 154: 365-374.

Gaykema, R.P., van der Kuil, J., Hersh, L.B., and Luiten, P.G. 1991. Pattern of direct projections from the hippocampus to the medial septum-diagonal band complex: Anterograde tracing with Phaseolus vulgaris leucoagglutinin combined with immunohistochemistry of choline acetyltransferase. Neuroscience 43: 349-360.

Gilbert, P.E. and Kesner, R.P. 2003. Localization of function within the hippocampus: The role of the dorsal CA3 subregion in paired-associate learning. Behav. Neurosci. 117: 1385-1394.

Gilbert, P.E. and Kesner, R.P. 2006. The role of dorsal CA3 hippocampal subregion in spatial working memory and pattern separation. Behav. Brain Res. 169: 142-149.

Gilbert, P.E., Kesner, R.P., and Lee, I. 2001. Dissociating hippocampal subregions: A double dissociation between the dentate gyrus and CA1. Hippocampus 11: 626-636.

Gold, A.E. and Kesner, R.P. 2005. The role of the CA3 subregion of the dorsal hippocampus in spatial pattern completion in the rat. Hippocampus 15: 808-814.

Hampson, R.E., Hedberg, T., and Deadwyler, S.A. 2000. Differential information processing by hippocampal and subicular neurons. Ann. N.Y. Acad. Sci. 911: 151-165.

Hargreaves, E.L., Rao, G., Lee, I., and Knierim, J.J. 2005. Major dissociation between medial and lateral entorhinal input to dorsal hippocampus. Science 308: 1792-1794.

Hasselmo, M.E. and McGaughy, J. 2004. High acetylcholine levels set circuit dynamics for attention and encoding and low acetylcholine levels set dynamics for consolidation. Prog. Brain Res. 145: 207-231.

Hasselmo, M.E. and Schnell, E. 1994. Laminar selectivity of the cholinergic suppression of synaptic transmission in rat hippocampal region CA1: Computational modeling and brain slice physiology. $J$. Neurosci. 14: 3898-3914.

Hasselmo, M.E. and Wyble, B.P. 1997. Free recall and recognition in a network model of the hippocampus: Simulating effects of scopolamine on human memory function. Behav. Brain Res. 89: $1-34$.

Hasselmo, M.E., Schnell, E., and Barkai, E. 1995. Dynamics of learning and recall at excitatory recurrent synapses and cholinergic modulation in rat hippocampal region CA3. J. Neurosci. 15: 5249-5262.

Hasselmo, M.E., Bodelon, C., and Wyble, B.P. 2002. A proposed function for hippocampal theta rhythm: Separate phases of encoding and retrieval enhance reversal of prior learning. Neural Comput. 14: 793-817.

Hoang, L. and Kesner, R.P. Dorsal hippocampus, CA3, and CA1 lesions disrupt temporal sequence completion. Behav. Neurosci. (in press).

Howard, M.W. and Natu, V.S. 2005. Place from time: Reconstructing 
position from a distributed representation of temporal context. Neural Netw. 18: 1150-1162.

Howard, M.W., Fotedar, M.S., Datey, A.V., and Hasselmo, M.E. 2005. The temporal context model in spatial navigation and relational learning: Toward a common explanation of medial temporal lobe function across domains. Psychol. Rev. 112: 75-116.

Hunsaker, M.R., Thorup, J.A., Welch, T., and Kesner, R.P. 2006. The role of CA3 and CA1 in the acquisition of an object-trace-place paired associate task. Behav. Neurosci. 120: 1252-1256.

Hunsaker, M.R., Allan, K.D., and Kesner, R.P. 2007a. The role of dCA3 efferents via the fimbria in the acquisition of a delay-non-match to place task. Hippocampus 17: 494-502.

Hunsaker, M.R., Rogers, J.L., and Kesner, R.P. 2007b. Behavioral characterization of a transection of dorsal CA3 subcortical efferents: Comparison with scopolamine and physostigmine infusions into dorsal CA3. Neurobiol. Learn. Mem. 88: 127-136.

Hunsaker, M.R., Mooy, G.G., Swift, J.S., and Kesner, R.P. 2007c. Dissociations of the medial and lateral perforant path projections into dorsal DG, CA3, and CA1 for spatial and nonspatial (visual object) information processing. Behav. Neurosci. 121: 742-750.

Jensen, O. and Lisman, J.E. 2005. Hippocampal sequence-encoding driven by a cortical multi-item working memory buffer. Trends Neurosci. 28: 67-72.

Jerman, T., Kesner, R.P., and Hunsaker, M.R. 2006. Disconnection analysis of CA3 and DG in mediating encoding but not retrieval in a spatial maze learning task. Learn. Mem. 13: 458-464.

Kesner, R.P. and Rolls, E.T. 2001. Role of long-term synaptic modification in short-term memory. Hippocampus 11: 240-250.

Kesner, R.P., Lee, I., and Gilbert, P. 2004 A behavioral assessment of hippocampal function based on a subregional analysis. Rev. Neurosci. 15: $333-351$

Kesner, R.P., Hunsaker, M.R., and Gilbert, P.E. 2005. The role of CA1 in the acquisition of an object-trace-odor paired associate task. Behav. Neurosci. 119: 781-786.

Kesner, R.P., Hunsaker, M.R., and Warthen, M.W. 2007. CA3 mediates arbitrary associations and episodic memory processing based on performance of reciprocal object place recall tasks. Soc. Neurosci. Abst. (in press).

Krug, M., Brödemann, R., and Wagner, M. 2001. Simultaneous activation and opioid modulation of long-term potentiation in the dentate gyrus and the hippocampal CA3 region after stimulation of the perforant pathway in freely moving rats. Brain Res. 913: 68-77.

Lee, I. and Kesner, R.P. 2002. Differential contribution of NMDA receptors in hippocampal subregions to spatial working memory. Nat. Neurosci. 5: $162-168$.

Lee, I. and Kesner, R.P. 2003. Differential role of dorsal hippocampus subregions in spatial working memory with short versus intermediate delay. Behav. Neurosci. 117: 1044-1053.

Lee, I. and Kesner, R.P. 2004a. Differential contributions of dorsal hippocampal subregions to memory acquisition and retrieval in contextual fear-conditioning. Hippocampus 14: 301-310.

Lee, I. and Kesner, R.P. 2004b. Encoding versus retrieval of spatial memory: Double dissociation between the dentate gyrus and the perforant path inputs into CA3 in the dorsal hippocampus. Hippocampus 14: 66-76.

Lee, I., Rao, G., and Knierim, J.J. 2004. A double dissociation between hippocampal subfields: Differential time course of CA3 and CA1 place cells for processing changed environments. Neuron 42: 803-815.

Lee, I., Jerman, T.S., and Kesner, R.P. 2005. Disruption of delayed memory for a sequence of spatial locations following CA1- or CA3-lesions of the dorsal hippocampus. Neurobiol. Learn. Mem. 84: $138-147$.

Leutgeb, J.K., Leutgeb, S., Treves, A., Meyer, R., Barnes, C.A., McNaughton, B.L., Moser, M.-B., and Moser, E.I. 2005. Progressive transformation of hippocampal neuronal representations in "morphed" environments. Neuron 48: 345-358.

Leutgeb, J.K., Leutgeb, S., Moser, M.-B., and Moser, E.I. 2007. Pattern separation in the dentate gyrus and CA3 of the hippocampus. Science 315: $961-966$.

Levy, W.B. 1996. A sequence predicting CA3 is a flexible association that learns and uses context to solve hippocampal-like tasks. Hippocampus 6: 579-590.

Li, X.-G., Somogyi, P., Ylinen, A., and Buzsaki, G. 1994. The hippocampal CA3 network: An in vivo intracellular labeling study. $J$. Comp. Neurol. 339: 181-208.

Lisman, J.E. 1999. Relating hippocampal circuitry to function: Recall of memory sequences by reciprocal dentate-CA3 interactions. Neuron 22: $233-242$.

Lisman, J.E. and Grace, A.A. 2005. The hippocampal-VTA loop: Controlling the entry of information into long-term memory. Neuron 46: 703-713.
Lisman, J.E. and Otmakhova, N.A. 2001. Storage, recall, and novelty detection of sequences by the hippocampus: Elaborating on the SOCRATIC model to account for normal and aberrant effects of dopamine. Hippocampus 11: 551-568.

Lorente de Nó, R. 1934. Studies on the structure of the cerebral cortex. II. Continuation of the study of the ammonic system. J. Psychol. Neurol. 46: 113-177.

Marr, D. 1971. Simple memory: A theory for archicortex. Proc. R. Soc. Lond. B. Biol. Sci. 262: 23-81.

Martinez, C.O., Do, V.H., Martinez Jr., J.L., and Derrick, B.E. 2002. Associative long-term potentiation (LTP) among extrinsic afferents of the hippocampal CA3 region in vivo. Brain Res. 940: 86-94.

McNaughton, B.L. and Morris, R.G.M. 1987. Hippocampal synaptic enhancement and information storage within a distributed memory system. Trends Neurosci. 10: 408-415.

Mehta, M.R., Barnes, C.A., and McNaughton, B.L. 1997. Experience-dependent, asymmetric expansion of hippocampal place fields. Proc. Natl. Acad. Sci. 94: 8918-8921.

Mehta, M.R., Lee, A.K., and Wilson, M.A. 2002. Role of experience and oscillations in transforming a rate code into a temporal code. Nature 417: 741-746.

Mizumori, S.J.Y., Perez, G.M., Alvarado, M.C., Barnes, C.A., and McNaughton, B.L. 1990. Reversible inactivation of the medial septum differentially affects two forms of learning in rats. Brain Res. 528: $12-20$

Mizumori, S.J., Ragozzino, K.E., Cooper, B.G., and Leutgeb, S. 1999 Hippocampal representational organization and spatial context Hippocampus 9: 444-451.

Morris, R. 2007. Theories of hippocampal function. In The hippocampus book (eds. P. Andersen et al.), pp. 581-658. Oxford University Press, Oxford.

Nakazawa, K., Quirk, M.C., Chitwood, R.A., Watanabe, M., Yeckel, M.F., Sun, L.D., Kato, A., Carr, C.A., Johnston, D., Wilson, M.A., et al. 2002. Requirement for hippocampal CA3 NMDA receptors in associative memory recall. Science 297: 211-218.

Nakazawa, K., Sun, L.D., Quirk, M.C., Rondi-Reig, L., Wilson, M.A., and Tonegawa, S. 2003. Hippocampal CA3 NMDA receptors are crucial for memory acquisition of one-time experience. Neuron 38: 305-315.

Olton, D.S., Shapiro, M.L., and Hulse, S.H. 1984. Working memory and serial patterns. In Animal cognition (eds. H.L. Roitblatt et al.), pp. 171-182. Lawrence Erlbaum Associates, Hillsdale, NJ.

O'Reilly, R.C. and McClelland, J.L. 1994. Hippocampal conjunctive encoding, storage, and recall: Avoiding a trade-off. Hippocampus 4: $661-682$.

O'Reilly, R.C. and Rudy, J.W. 2001. Conjunctive representations in learning and memory: Principles of cortical and hippocampal function. Psychol. Rev. 108: 311-345.

Pereira, P.M., Cosquer, B., Schimchowitsch, S., and Cassel, J.-C. 2005 Hebb-Williams performance and scopolamine challenge in rats with partial immunotoxic hippocampal cholinergic deafferentation. Brain Res. Bull. 64: 381-394.

Poucet, B. 1989. Object exploration, habituation, and response to a spatial change in rats following septal or medial frontal cortical damage. Behav. Neurosci. 103: 1009-1016.

Raisman, G., Cowan, M.W., and Powell, T.P.S. 1966. An experimental analysis of the efferent projection of the hippocampus. Brain 89: 83-108.

Rajji, T., Chapman, D., Eichenbaum, H., and Greene, R. 2006. The role of CA3 hippocampal NMDA receptors in paired associate learning. $J$. Neurosci. 26: 908-915.

Risold, P.Y. and Swanson, L.W. 1997. Connections of the rat lateral septal complex. Brain Res. Rev. 24: 115-195.

Rogers, J.L. and Kesner, R.P. 2003. Cholinergic modulation of the hippocampus during encoding and retrieval. Neurobiol. Learn. Mem. 80: $332-342$.

Rogers, J.L. and Kesner, R.P. 2004. Cholinergic modulation of the hippocampus during encoding and retrieval of tone/shock-induced fear conditioning. Learn. Mem. 11: 102-107.

Rolls, E.T. 1989a. The representation and storage of information in neuronal networks in the primate cerebral cortex and hippocampus. In The computing neuron (eds. R. Durbin et al.), pp. 125-159. Addison-Wesley, Wokingham, UK.

Rolls, E.T. 1989b. Functions of neuronal networks I the hippocampus and neocortex in memory. In Neural models of plasticity: Experimental and theoretical approaches (eds. J.H. Bryne and W.O. Berry), pp. 240-265. Academic Press, San Diego.

Rolls, E.T. 1996. A theory of hippocampal function in memory. Hippocampus 6: 601-620.

Rolls, E.T. and Kesner, R.P. 2006. A computational theory of hippocampal function, and empirical tests of the theory. Prog. Neurobiol. 79: 1-48.

Rolls, E.T. and Treves, A. 1998. Neural networks and brain function. 
Oxford University Press, Oxford.

Rolls, E.T., Xiang, J.-Z., and Franco, L. 2005. Object, space and object-space representations in the primate hippocampus. $J$. Neurophysiol. 94: 833-844.

Samsonovich, A. and McNaughton, B.L. 1997. Path integration and cognitive mapping in a continuous attractor neural network model. J. Neurosci. 17: 5900-5920.

Scharfman, H.E. 2007. The CA3 "backprojection" to the dentate gyrus. Prog. Brain Res. 163: 627-637.

Swanson, L.W. and Cowan, W.M. 1977. An autoradiographic study of the organization of the efferent connections of the hippocampal formation of the rat. J. Comp. Neurol. 172: 49-84.

Swanson, L.W. and Cowan, W.M. 1979. The connections of the septal region in the rat. J. Comp. Neurol. 186: 621-656.

Tanila, H. 1999. Hippocampal place cells can develop distinct representations of two visually identical environments. Hippocampus 9: $235-246$.

Treves, A. and Rolls, E.T. 1994. Computational analysis of the role of the hippocampus in memory. Hippocampus 4: 374-391.

Vago, D.R., Bevan, A., and Kesner, R.P. 2007. The role of the direct perforant path input to the CA1 subregion of the dorsal hippocampus in memory retention and retrieval. Hippocampus 17: 977-987.
Vann, S.D. and Aggleton, J.P. 2004. The mammillary bodies: Two memory systems in one? Nat. Rev. Neurosci. 5: 35-44.

Vazdarjanova, A. and Guzowski, J.F. 2004. Differences in hippocampal neuronal population responses to modifications of an environmental context: Evidence for distinct, yet complementary, functions of CA3 and CA1 ensembles. J. Neurosci. 24: 6489-6496.

Vinogradova, O.S., Brazhnik, E.S., Kichigina, V.F., and Stafekhina, V.S. 1996. Modulation of the reaction of hippocampal neurons to sensory stimuli by cholinergic substances. Neurosci. Behav. Physiol. 26: 113-124.

Wirth, S., Yanike, M., Frank, L.M., Smith, A.C., Brown, E.N., and Suzuki, W.A. 2003. Single neurons in the monkey hippocampus and learning of new associations. Science 300: 1578-1581.

Witter, M.P., Van Hoesen, G.W., and Amaral, D.G. 1989. Topographical organisation of the entorhinal projection to the dentate gyrus of the monkey. J. Neurosci. 9: 216-228.

Wyss, X., Swanson, I.W., and Cowan, W.M. 1980. The organization of the fimbria, dorsal fornix, and ventral hippocampal commissure in the rat. Anat. Embryol. (Berl.) 138: 303-316.

Received July 2, 2007; accepted in revised form August 31, 2007. 


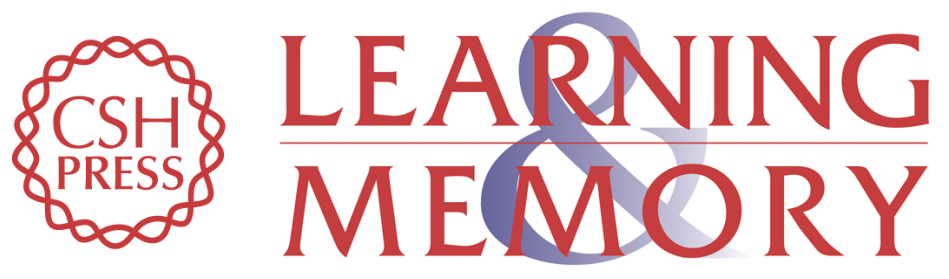

\section{Behavioral functions of the CA3 subregion of the hippocampus}

Raymond P. Kesner

Learn. Mem. 2007, 14:

Access the most recent version at doi:10.1101//m.688207

References This article cites 81 articles, 14 of which can be accessed free at: http://learnmem.cshlp.org/content/14/11/771.full.html\#ref-list-1

License

Email Alerting Receive free email alerts when new articles cite this article - sign up in the box at the Service top right corner of the article or click here. 